\title{
Research Paper \\ The Effect of Shoe Type and Load Carrying on Electromyographic Activity of Lower Extremity Muscles during Stair Ascent and Descent
}

\author{
*Nader Farahpour ${ }^{1}$, Mahdi Majlesi ${ }^{2}$, Mohammad Reza Hoseinpouri ${ }^{3}$ \\ 1. Department of Sport Biomechanics, Faculty of Physical Education and Sport Sciences, Bu Ali Sina University, Hamedan, Iran. \\ 2. Department of Physical Education and Sport Sciences, Faculty of Humanities, Hamedan Branch, Islamic Azad University, Hamedan, Iran. \\ 3. Department of Physical Education and Sport Sciences, Faculty of Humanities, Boroujerd Branch, Islamic Azad University, Boroujerd, Iran.
}

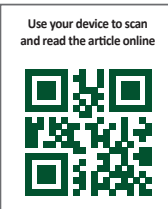

Citation: Farahpour N, Majlesi M, Hoseinpouri M. [The Effect of Shoe Type and Load Carrying on Electromyographic Activity of Lower Extremity Muscles during Stair Ascent and Descent (Persian)]. Journal of Sport Biomechanics. 2019; 5(2):92-101. https://doi.org/10.32598/biomechanics.5.2.2

https://doi.org/10.32598/biomechanics.5.2.2

Key words:

Load carrying, Shoes,

Stair Ascent and De-

scent, Muscle activity

\section{ABSTRACT}

Objective Stair ascent and descent is an essential movement task in daily life in which individuals are subjected to repetitive impact forces. The purpose of this study was to evaluate the intensity of Electromyographic (EMG) activity in lower extremity muscles of healthy young men during stair ascent and descent task wearing different type of shoes and carrying loads.

Methods Nine men with a mean age of $25.94 \pm 3.26$ years, mean height of $174 \pm 7.4 \mathrm{~cm}$, and mean weight of $70.95 \pm 8.25 \mathrm{~kg}$ were selected. Four stairs were fabricated and the electromyographic activity of their lower extremity muscles (two muscles in the posterior leg and three quadriceps muscles) in the right side of the body was measured using the 16-channel EMG system MA300 during the task. These tests were conducted in two conditions of with and without load carrying. The load was a cube-shaped box weighing $15 \%$ of the body weight. Three cases of footwear were set: barefoot, athletic shoes, and formal shoes. Repeated measure ANOVA was used for data analysis at the significant level of $\mathrm{P}<0.05$.

Results The load factor had a significant effect on the intensity of muscle activity. The intensity of muscle activity during ascending stairs was higher than that during descending. In stair descent task, the EMG activity of the vastus medialis muscle was greater than that of the vastus lateralis and rectus femoris muscles, which causes the patella to be pulled inward more leading to patellofemoral articular cartilage wear in the long term.

Conclusion Stair ascent puts more pressure on the ankle and knee joints. When carrying the load up stairs, the use of proper shoes can greatly reduce the intensity of muscle activity and delay fatigue. It is, therefore, recommended that people with patellofemoral articular cartilage wear should not use the stairs, if possible.

\section{Extended Abstract}

\section{Introduction}

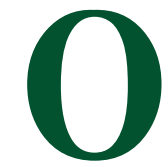

ne of the most common daily motor tasks is stair ascent and descent walk, which requires more muscle activity and range of motion in the joints of the lower limbs than normal walking [1]. In walking up and down stairs, in addition to applying force to move forward, more force is needed to move the body upward, where the knee and ankle muscles are responsible for creating this force when ascending [2, 3]. During walking, the joints are exposed to repeated impact forces at the moment the heel makes contact with the ground, which

* Corresponding Author:

Nader Farahpour, PhD.

Address: Department of Sport Biomechanics, Faculty of Physical Education and Sport Sciences, Bu Ali Sina University, Hamedan, Iran.

Tel: +98 (918) 1113816

E-mail: naderfarahpour1@gmail.com 
over time can lead to destructive changes in them. These impact forces during walking on stairs, especially when descending, can be even more severe [1]; so, a suitable shoe can reduce these impact forces and their effect. The choice of footwear is different between people. Some prefer to wear formal shoes with relatively hard heels; some prefer to wear shoes with high heels, and others prefer sneakers. The type of shoe can affect muscle activity and postural alignment, the distribution of force on the sole of the foot, and the ground reaction force, and if not suitable, it can lead to clinical problems in the lower extremity [10].

So far, several studies have been performed on the kinematics, kinetics, and electromyography of muscles when walking up and down stairs [13-16]. The electromyographic activity of the lower extremity muscles while walking on stairs can provide an overview of how these muscles and the forces applied to the lower extremity joints work. When climbing stairs, a lot of pressure is put on the knee joint, which is caused by the contraction of the quadriceps muscles [17]. The present study aimed to examine the effect of show type and load carrying on Electromyographic (EMG) activity of lower extremity muscles during stair ascent and descent walk.

\section{Participants and Methods}

In this quasi-experimental study, from among male students of Islamic Azad University, Hamedan Branch, 9 aged 20-30 years were randomly and voluntarily selected after ensuring their general health and no history of any injuries, including fracture in limbs, especially lower limbs and spine. A digital scale and a stadiometer (Seca model), were used to measure the weight and height of the subjects, respectively. The 16-channel EMG system (MA 300, Motion Lab Systems, US) was used to record the activity of internal

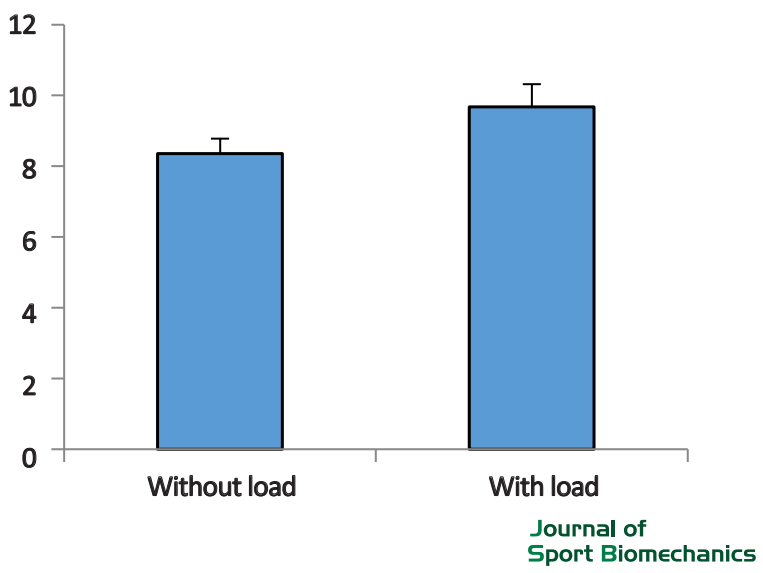

Figure 1. Comparing the effect of load on EMG activity of lower extremity muscles and external twin muscles, the vastus medialis, vastus lateralis, and rectus femoris muscles. These points were selected according to McGill (1996)'s protocol. After installing the electrodes, Maximal Voluntary Isometric Contraction (MVIC) of the muscles was performed to normalize the data. The participants were first tested without load carrying and performing the stair ascent and descent task with bare feet. Then, it was done with the load (carrying a box weighing $15 \%$ of the body weight) and performing the task once wearing athletic shoes and once with formal shoes. Subjects were asked to use the most convenient form and the usual speed to complete the task.

\section{Results}

The results showed that the load factor significantly increases the intensity of muscle activity (Figure 1) indicating that carrying loads during stair ascent and descent task significantly increases EMG activity of lower extremity muscles $(\mathrm{F}=25.2, \mathrm{P}=0.0001)$. Moreover, the intensity of EMG activity during ascending and descending stairs was significantly different. As shown in Figure 2, its intensity in ascending was higher than in descending $(\mathrm{F}=58.01$, $\mathrm{P}=0.0001$ ). Regarding the shoe factor, results showed that the intensity of EMG activity in the three cases of barefoot, athletic shoes and formal shoes did not differ significantly $(\mathrm{F}=2.86, \mathrm{P}=0.08)$. Factor analysis showed that the interaction between the two factors of load and direction (ascending and descending) is not significant; this means that the effect of load carrying on the intensity of EMG activity during ascending and descending stairs was similar. Furthermore, the intensity of EMG activity in different muscles changed equally in both conditions of with and without load carrying $(\mathrm{F}=1.53, \mathrm{P}=0.35)$; i.e., although the intensity of EMG activity under load carrying was increased significantly, but it increased the intensity of EMG activity in all muscles.

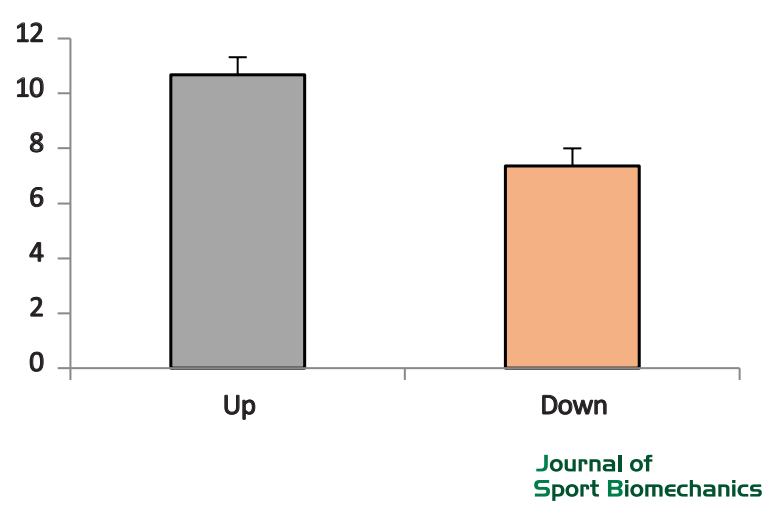

Figure 2. Comparing the effect of direction on EMG activity of lower extremity muscles 


\section{Discussion}

The load factor has a significant effect on EMG activity of lower extremity muscles; i.e., the intensity of EMG activity increases by load carrying. The direction factor (ascending and descending) has also a significant effect on EMG activity of muscles, where its intensity during ascending stairs increased by about $45 \%$ compared to its intensity when descending. In addition to increased intensity, the pattern of EMG activity varied in both directions where the activity of internal and external twin muscles as well as vastus medialis and vastus lateralis muscles during climbing the stairs increased while the activity of rectus femoris muscle was reduced.

\section{Conclusion}

Based on these results, it can be said that, by descending the stairs, since the intensity of activity in vastus medialis muscle increases more than in vastus lateralis muscle, it causes the patella to be pulled inward more leading to the abrasion of the internal cartilage of patellofemoral joint in the long term. Therefore, it can be concluded that stair ascent and descent can be considered as a risk factor for osteoarthritis.

\section{Ethical Considerations}

\section{Compliance with ethical guidelines}

All subjects participated in the present study voluntarily after signing a consent form.

Funding

This research did not receive any specific grant from funding agencies in the public, commercial, or not-forprofit sectors.

Authors' contributions

All authors contributed equally in preparing this article.

Conflicts of interest

The authors declared no conflict of interest 


\title{
تأثير نوع كفش و حمل بار بر فعاليت الكتروميوتحر افى عضلات اندام تحتانى هنَّام بالا رفتن و يايين آمدن از بله
}

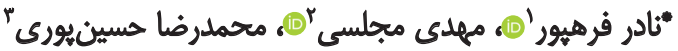

1. كروه بيومكانيك ورزشى، دانشكده تربيتبدنى و علوم ورزشى، دانشُكاه بوعلى سينا، همدانئ ايران.

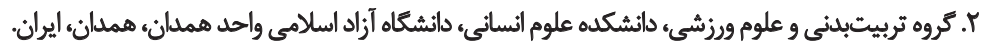

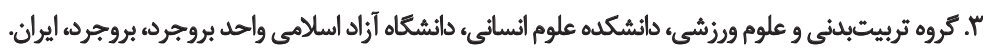

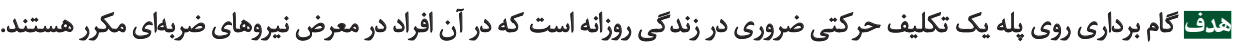

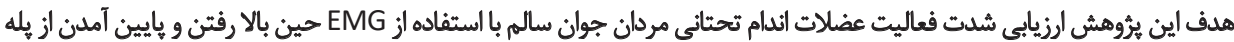

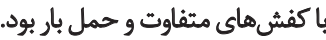

اطلاعات مقاله: تاريخ دريافت: rادى

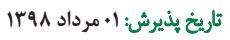

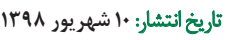

كليدوأرها:

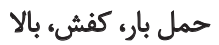

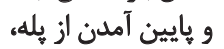
فعاليت عضلانى

مخرب در آنها شوند. اين بارهاى ضربهاى در كامبردارى روى

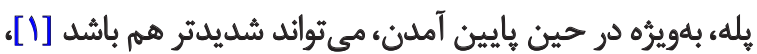

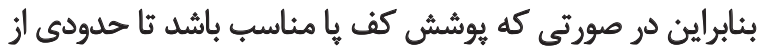

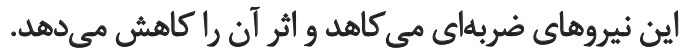
مطالعات قبلى نشان دادهاند كه در بالا و يايين رفتن از يله،

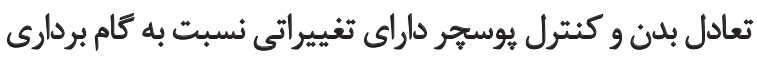

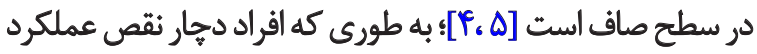

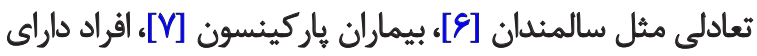

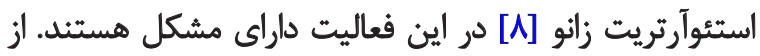

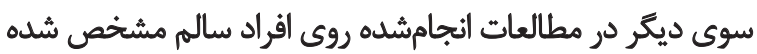

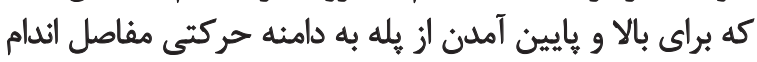

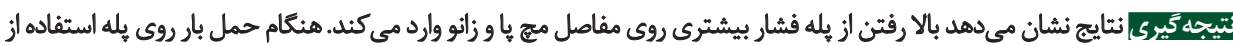

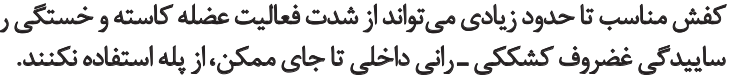

يكي از متداول ترين تكاليف حركتي روزمره بالا و هايين رفتن

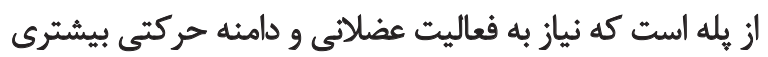

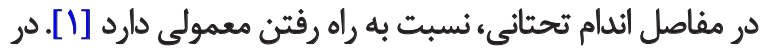

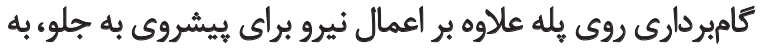

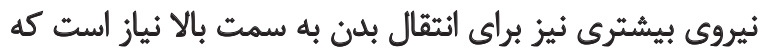

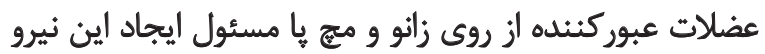

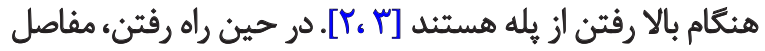

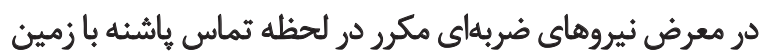

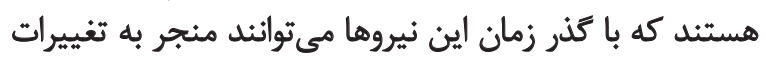

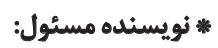

نادر فرهيور نشائي: هملان، دانشكاه آزاد اسلامى واحد همدان، دانشكده علوم أساني، كروه بيومكانيك ورزشى. تلغن: يست الكترونيكي: naderfarahpour1@gmail.com 
فعاليت الكتروميوكَافى عضلات اندام تحتانى هنكَام كامبردارى روى يله است.

$$
\text { روش مشناسى }
$$

در اين ثروهش نيمهتجربى از ميان دانشجويان مرد دانشكاه

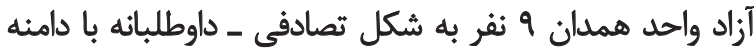

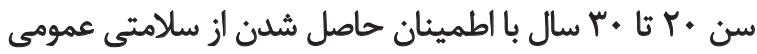

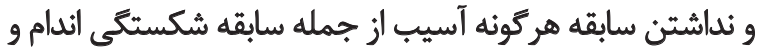

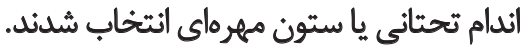
از ترازوى ديجيتال، قدسنج Seca براى اندازهكيرى وزن و قد آزمودنىها و از دستَّاه 9 اكاناله EMG مدل (Motion Lab System عضلانى اندام تحتانى استفاده شد.

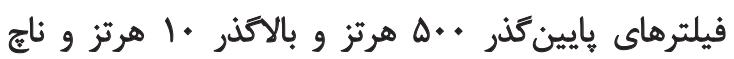

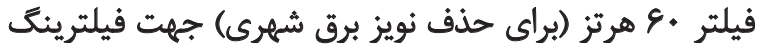

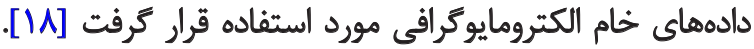

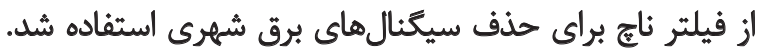

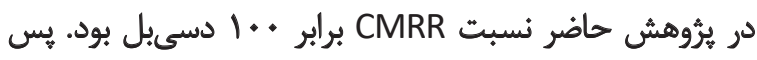

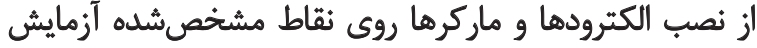

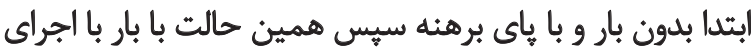

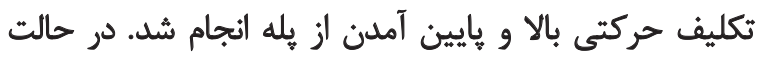

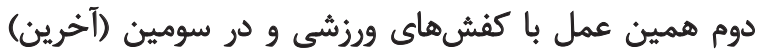

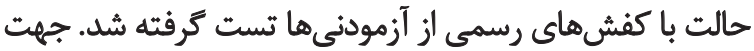

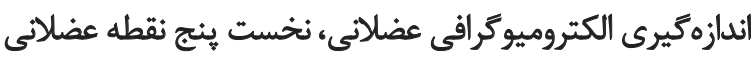

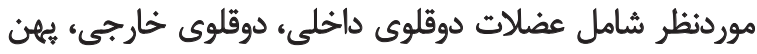

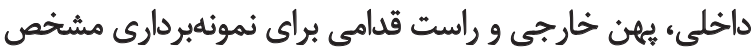

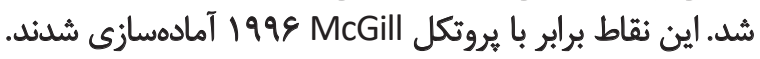

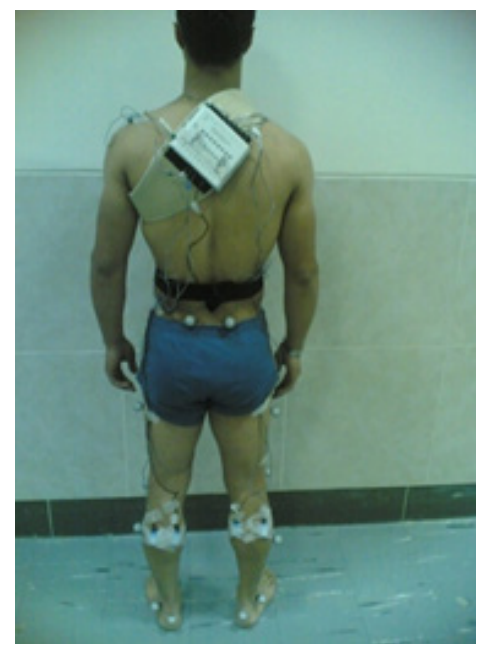

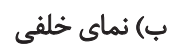

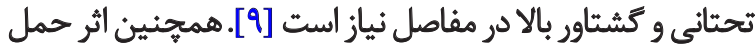

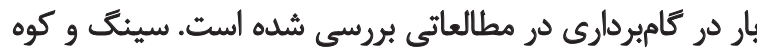

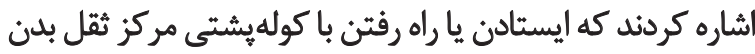

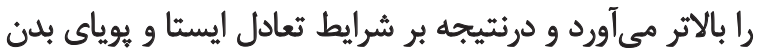

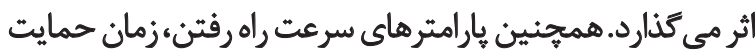

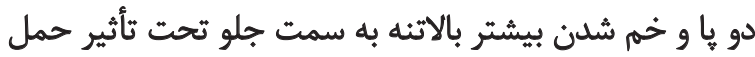

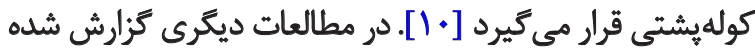

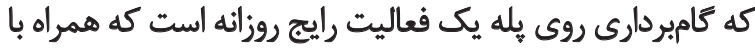

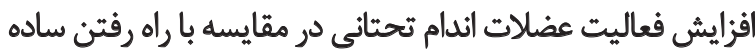

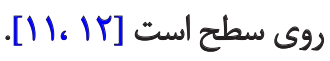

سليقه افراد در انتخاب نوع كفش متفاوت است، بعضى إز افراد

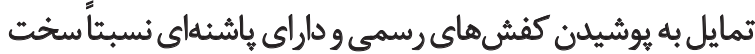

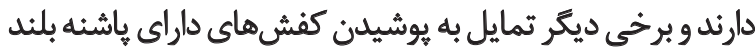

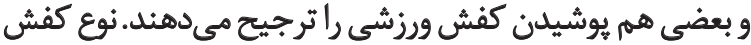

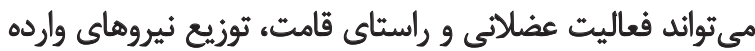

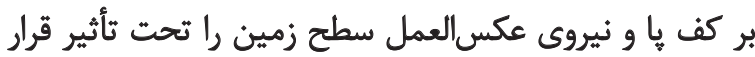

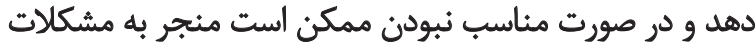

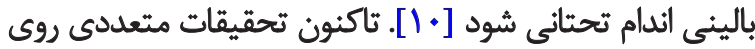

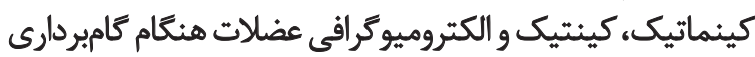

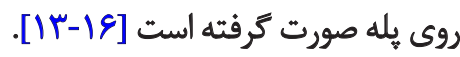
فعاليت الكتروميوگرافي عضلات اندام تحتانى حين كامبرداري

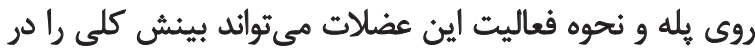

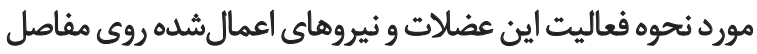

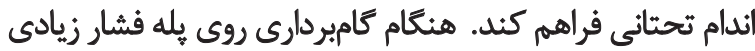

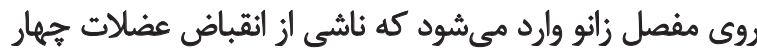

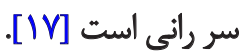
هدف از ئروهش حاضر بررسى اثر نوع كفش و حمل بار روى

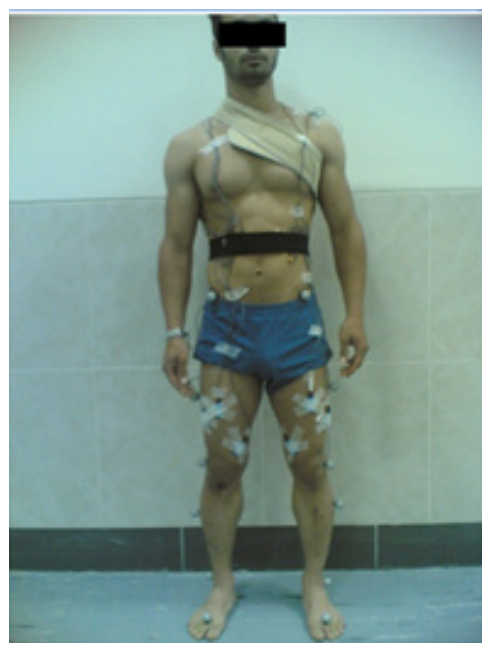

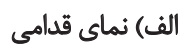

تصوير ا. نحوه نصب الكترودها و ماركرها 

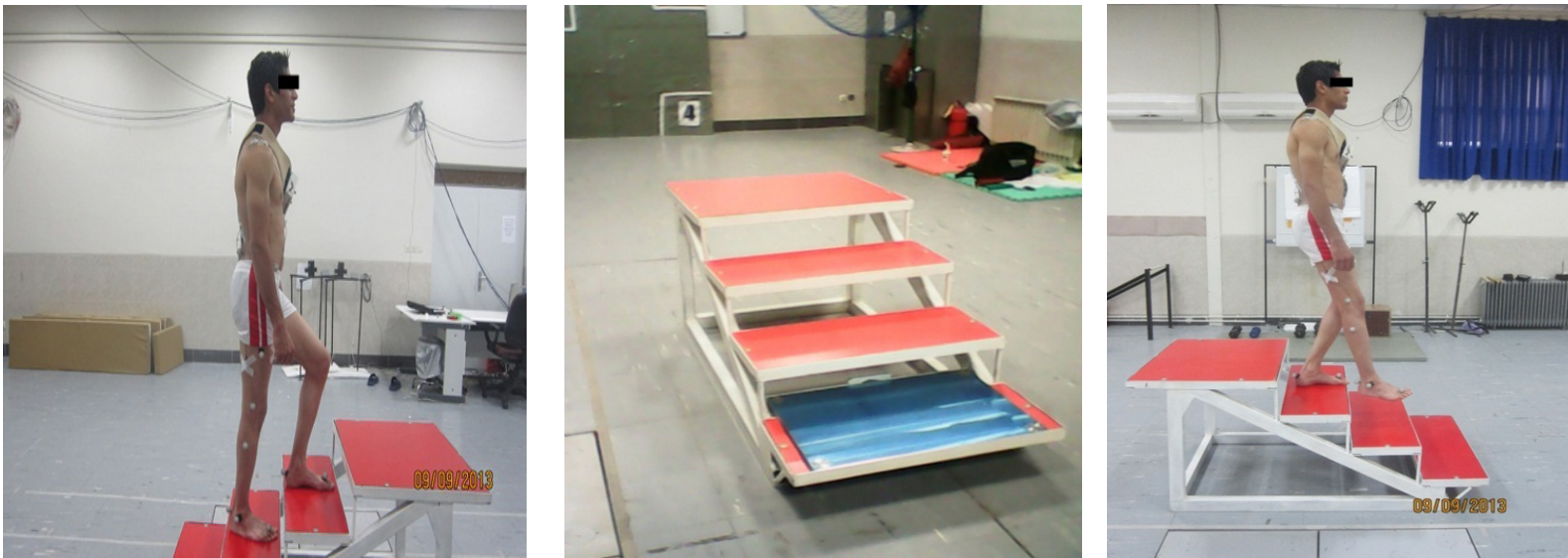

مجله بيومكانيك وزنش

تكليف به كار ببرند. هر آزمودنى تكليف حركتى بالارفتن و هايين

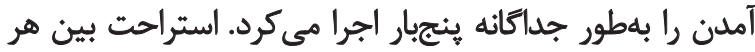
تكرار • ا ثانيه در نظر كرفته شد.

نتايج

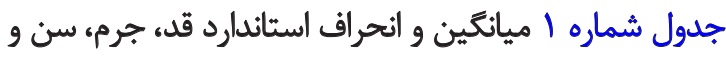

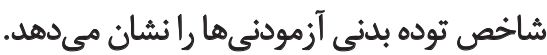

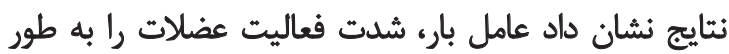

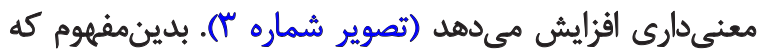

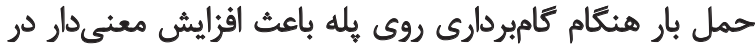

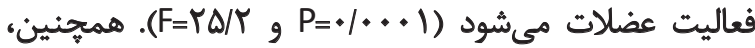

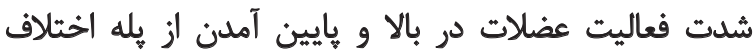

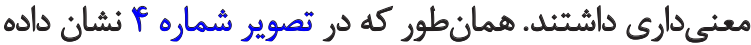

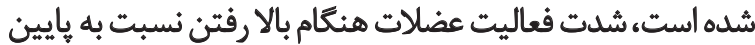

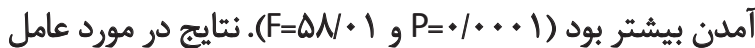

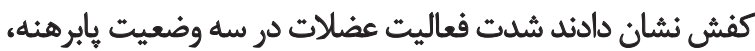

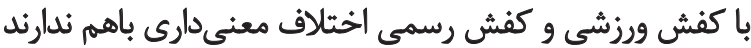
( $F=r / \Lambda \&, P=\cdot 1 \cdot \Lambda)$

تجزيه و تحليل عاملى نشان داد تعامل بين دو فاكتور بار و

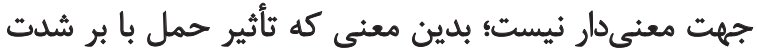

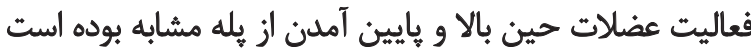

تصوير r. تكليف بالا رفتن و بايين آمدن بدون حمل بار

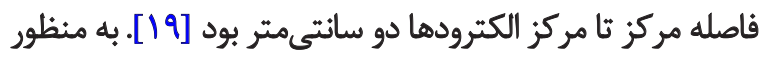

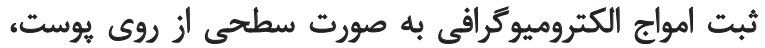

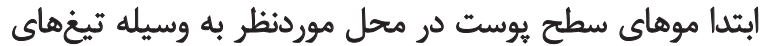

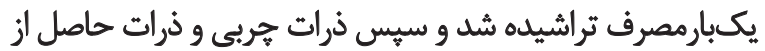

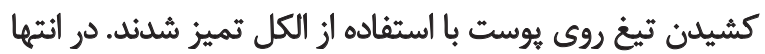

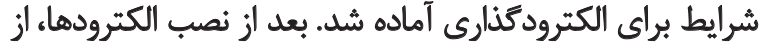

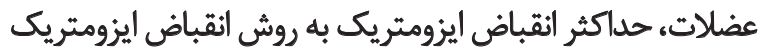

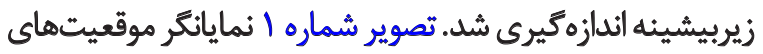
آناتوميكى انتخابشده جهت استقرار الكترودهاست.

مقادير انقباض زير بيشينه ايزومتريك به منظور نرمال سازي

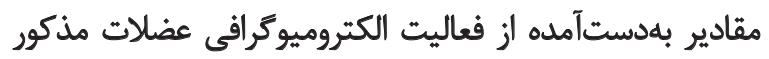

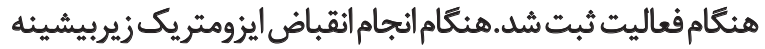

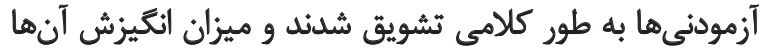
قبل از اجراى حركات موردنظر بالا برده شد.

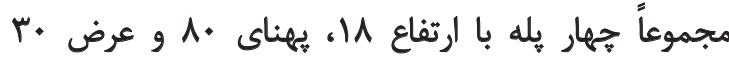

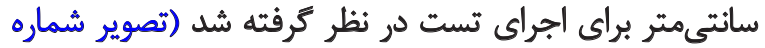

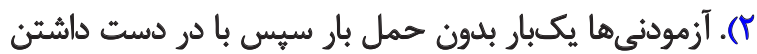

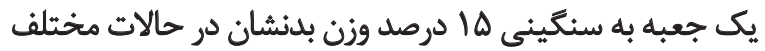

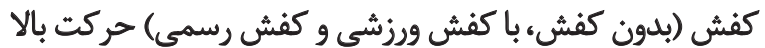

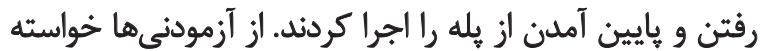

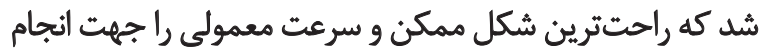

جدول ا. ميانكين و انحراف استاندارد قد، وزن، سن و شاخص توده بدنى آزمودنيها

\begin{tabular}{|c|c|}
\hline ميانئين土|نحراف استاندارد & متغير هاى أنترويومترى \\
\hline 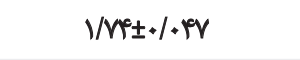 & قـد \\
\hline$V \cdot / 9 \Delta \pm N T \Delta$ & جرم \\
\hline$r \Delta / q Y \pm T / T E$ & سن \\
\hline$r \cdot 1 F q \pm N \cdot 8 N$ & شاخص توده بلدنى \\
\hline
\end{tabular}

مجله بيومكانيك ورنش 


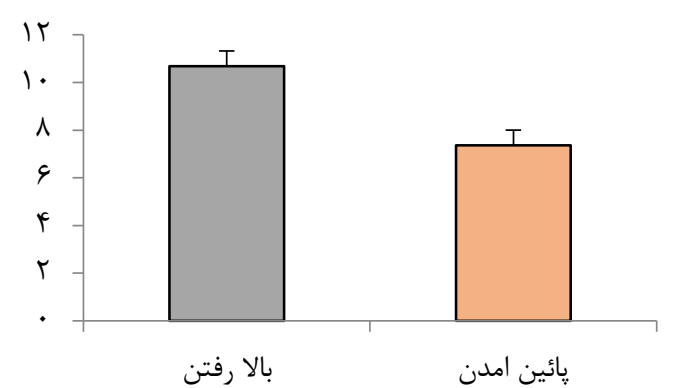

مجله بيومكانيك ولشن

تصوير F. اثر عامل جهت روى شدت فعاليت عضلات

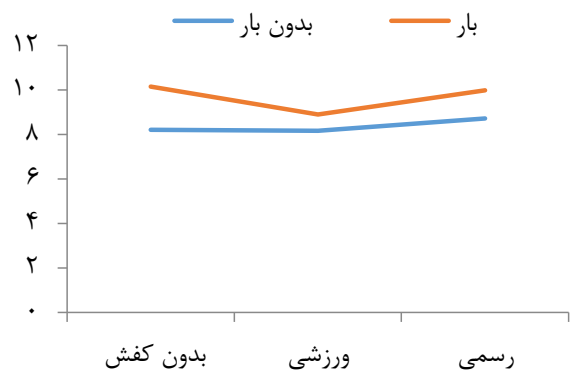

مجله بيومكانيك ولنث

$$
\text { تصوير و. تعامل بين عامل بار و كفش }
$$

نتايج تحليل عاملى نشان داد كه شدت فعاليت در عضلات

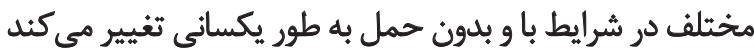

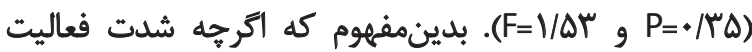

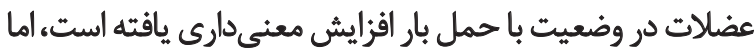

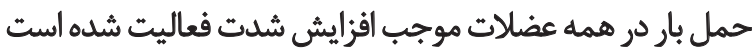

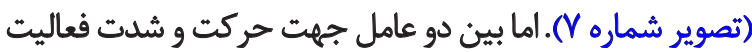

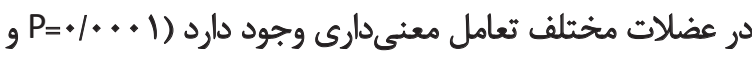
F=q/VD

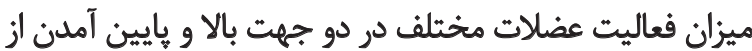
يله، با يكديكر متفاوت است.

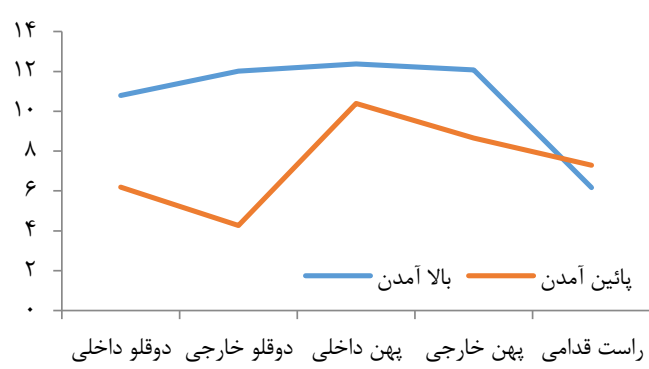

مجله بيومكانيك وزنث

تصوير A. تعامل بين جهت وعضله

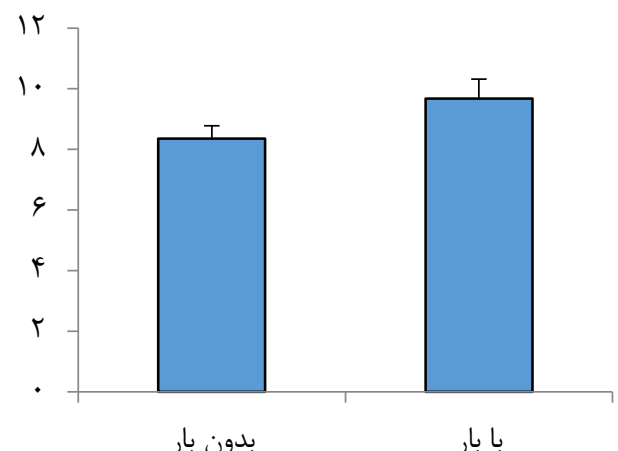

مجله بيومكانيك ورنث

تصوير ب. اثر عامل بار روى شدت فعاليت عضلات

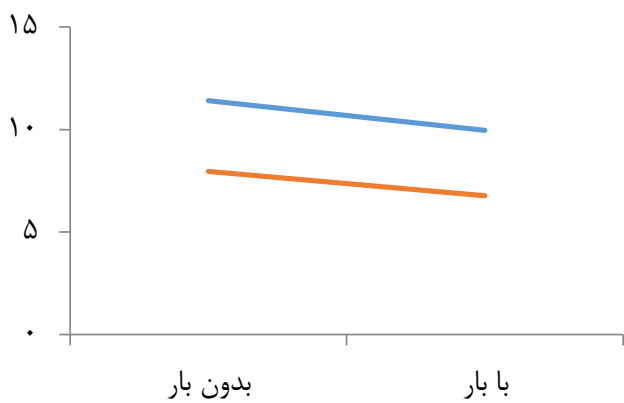

مجله بيومكانيك ولنش بارن

تصوير هـ تعامل بين عامل بار و جهيت

كه الين نتايج در تصوير شماره ه هشاهله هي شيود. همان كونه

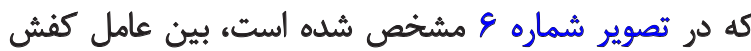

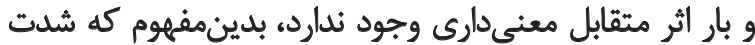

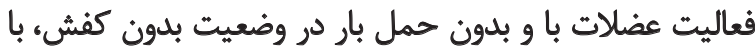

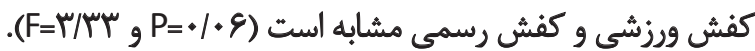

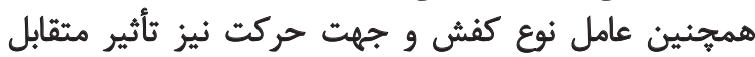

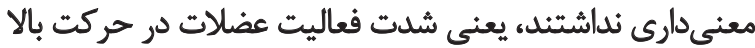

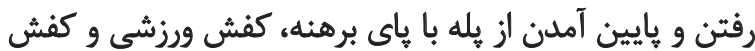

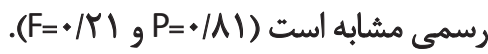

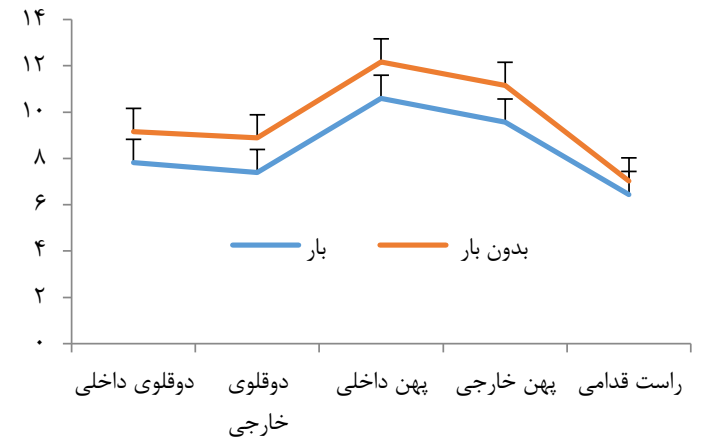

مجله بيومكانيك ولنث تصوير V. تعامل بين بار و عضله 


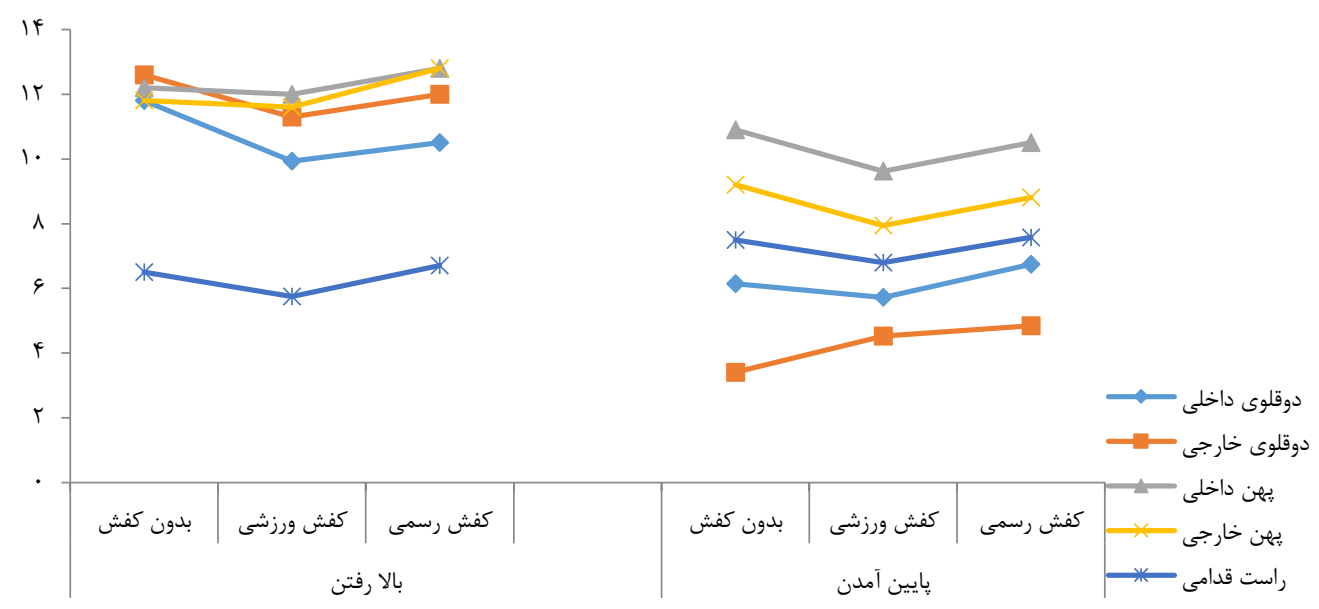

مجله بيومكانيك ورنثـ

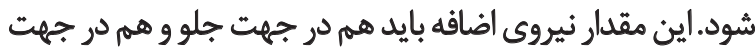

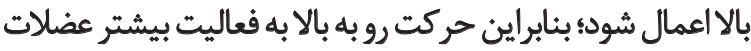

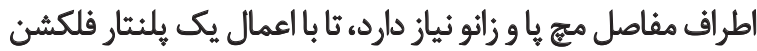

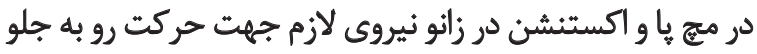

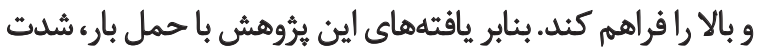

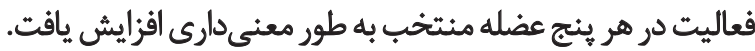

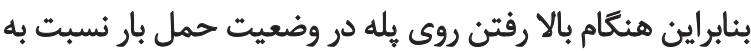

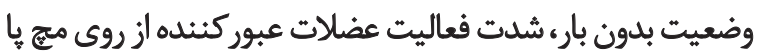
و عضلات عمل كننده روى زانو به مقدار مشابهى افزايش مى يابد با توجه به يافتههاى اين تحقيق مشاهده مي إشود كه عامل

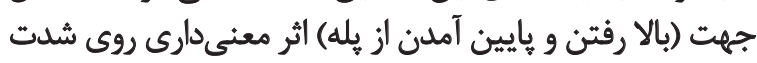

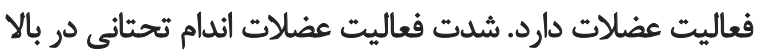

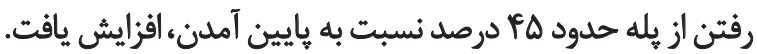

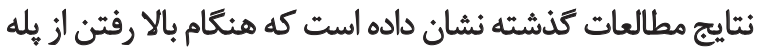

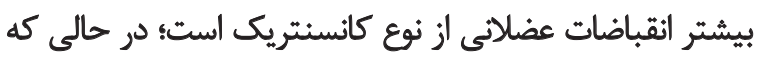

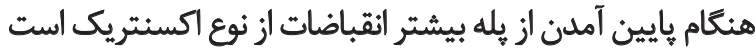

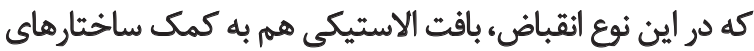

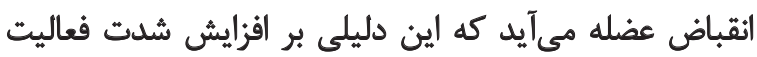

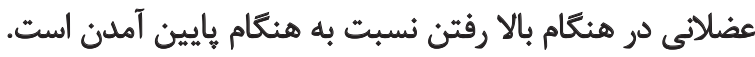

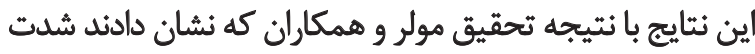

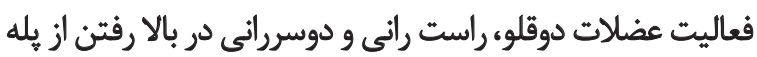

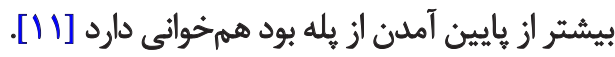

علاوه بر افزايش شدت فعاليت در بالاو و إيين آمدن از يله،

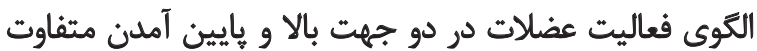

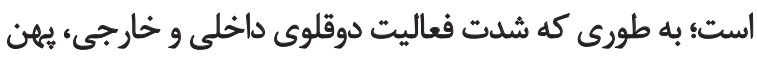

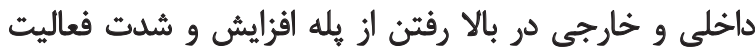

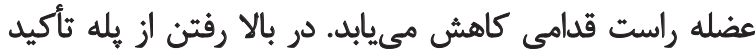

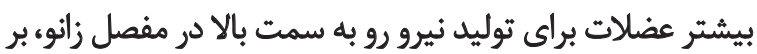

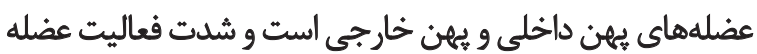

$$
\text { تصوير 9. تعامل معنى دار بين جهت، كفش و عضله }
$$

نتايج نشان داد عامل هاى جهت، كفش و عضله داراى تأثير

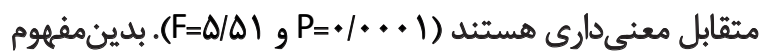

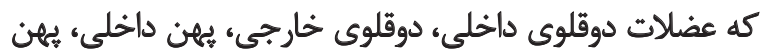

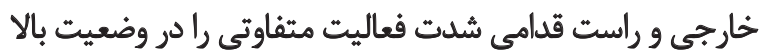

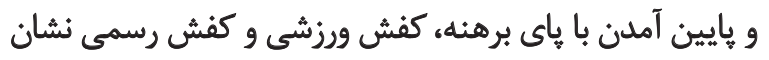

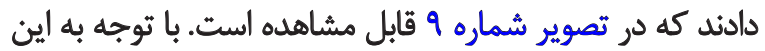

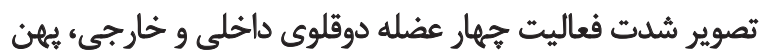

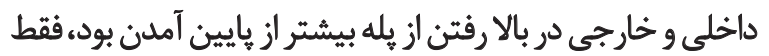

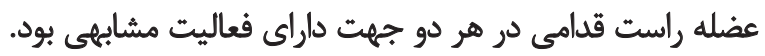

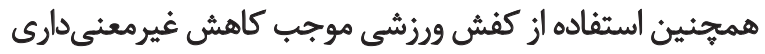
در شدت فعاليت عضلات مي استود.

بحث حركت روى يله از جمله فعاليتهاى است كه در طول شبانهروز

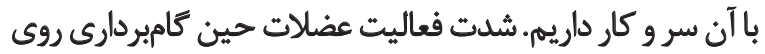

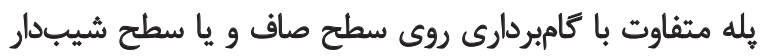

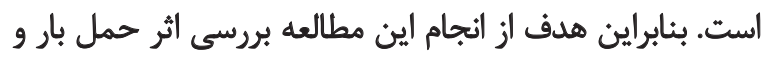

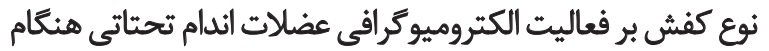
كامبردارى روى يله بود.

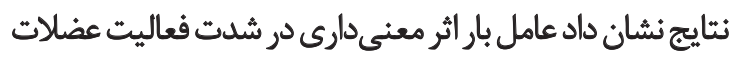

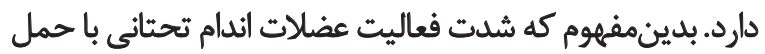

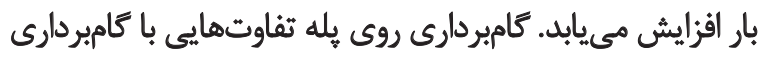

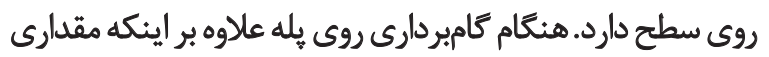

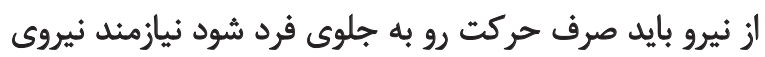

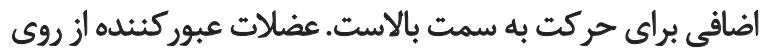

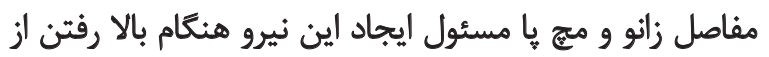

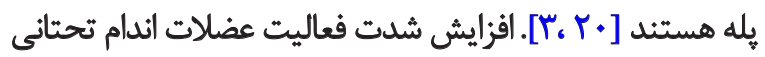

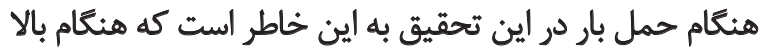

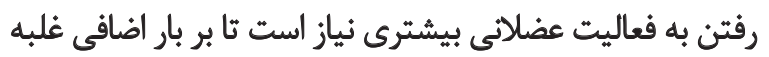


خارجى شدت فعاليت بيشترى نسبت به ديكر وضعيتها داشتند.

\section{نتيجليَيرىنهايي}

نتايج يُروهش حاضر نشان داد عامل بار اثر معنى دارى در شدت

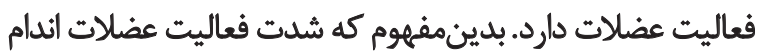

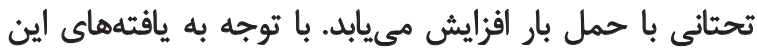

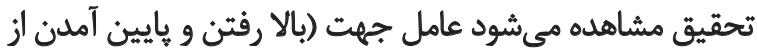

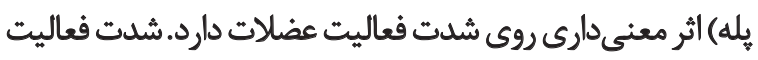

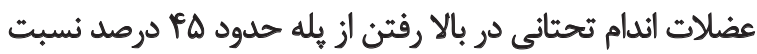

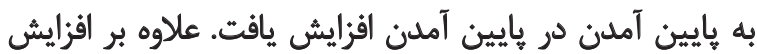

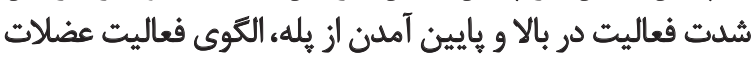

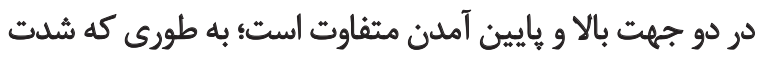

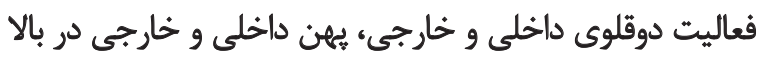

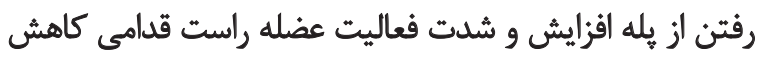

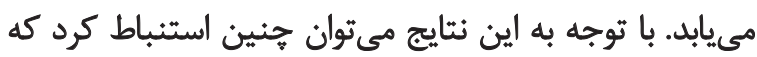

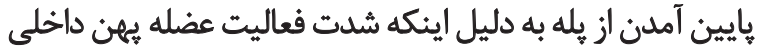

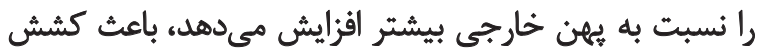

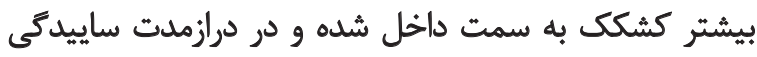

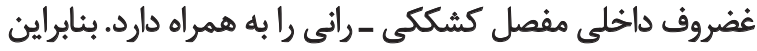

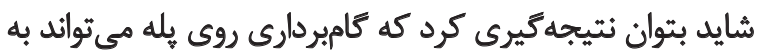

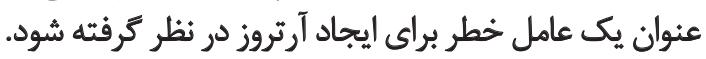

مالاحظات اخلاقى

\section{ييروى از اصول اخلاق يؤهش}

تمامى آزمودنىها به طور داوطلبانه و با تكميل رضايتنامه

شخصى در تحقيق حاضر شركت داشتند.

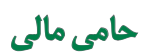

اين تحقيق هيج كمك مالى خاصى از سازمانهاى عمومى يا خصوصى دريافت نكرده است.

$$
\text { مشاركت نويسند متان }
$$

همه نويسندكان در آمادهازى مقاله مشاركت داشتهاند.

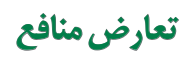

بنابر اظهار نويسند انان اين مقاله تعارض منافع ندارد.
راست قدامى كمتر است؛ در نتيجه در بالا رفتن از يله تماس

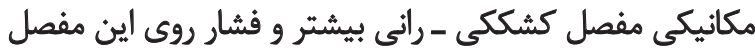

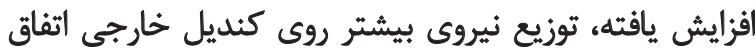

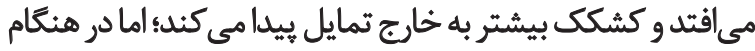

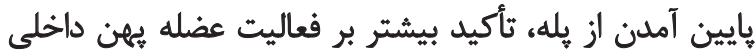

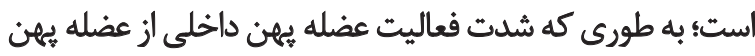

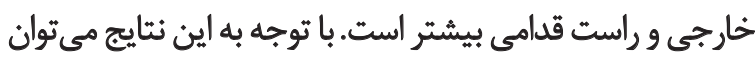

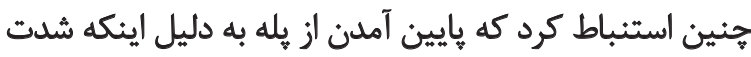

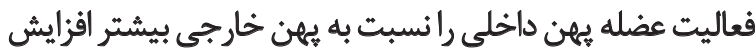

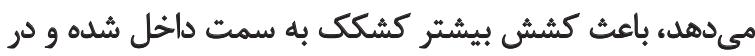

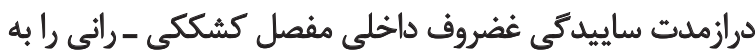

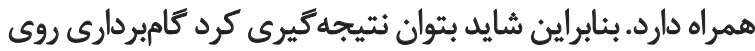

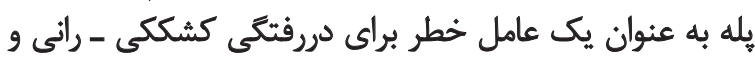

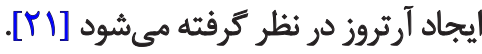

نتايج نشان داد عامل كفش اثر معنى دارى روى شدت كلى كلى ندانى

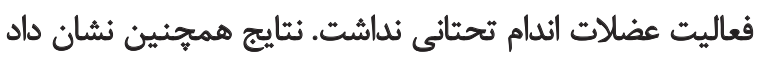

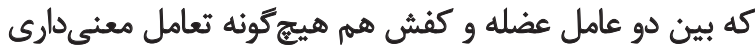

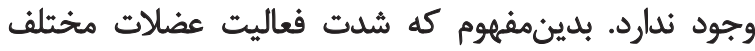

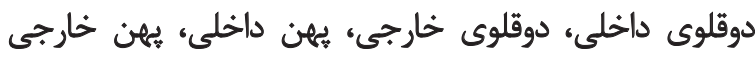

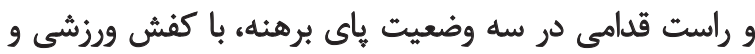

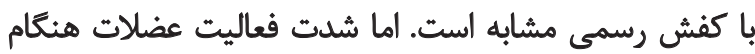

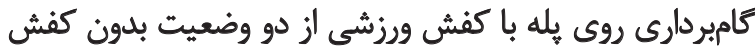

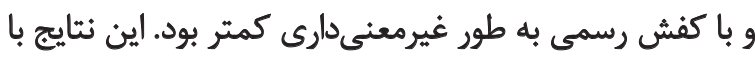

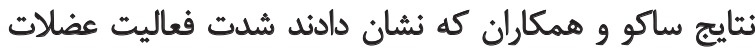

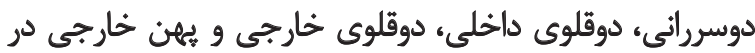

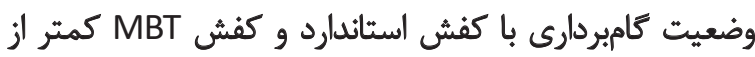

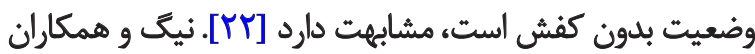

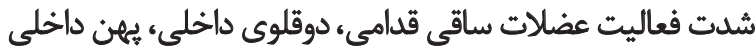

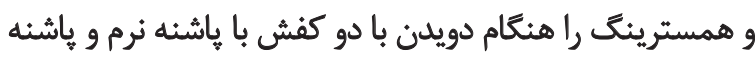

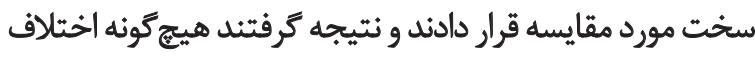

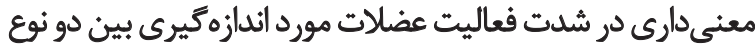

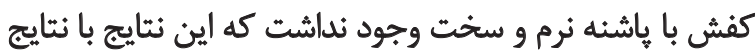

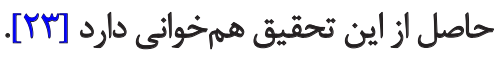

تجزيه و تحليل نتايج نشان داد بين عاملهاى بار، جهت و وارت

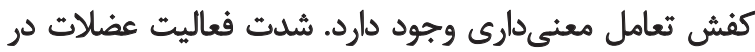

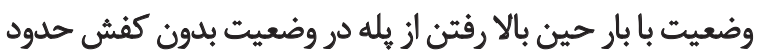

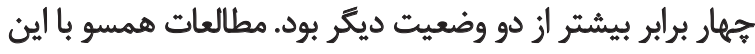

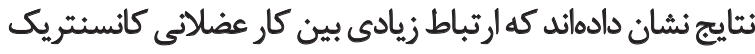

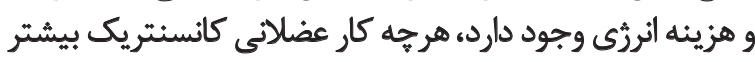

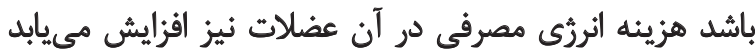

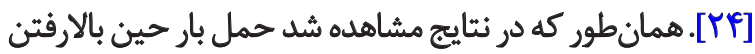

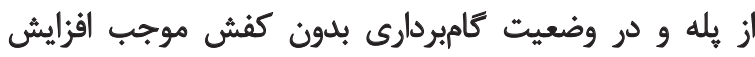

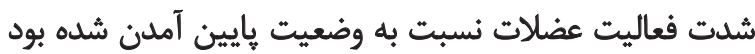

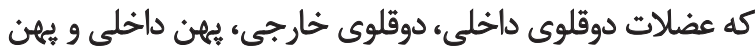




\section{References}

[1] Andriacchi TP, Andersson GB, Fermier RW, Stern D, Galante JO. A study of lower-limb mechanics during stair-climbing. The Journal of Bone \& Joint Surgery. 1980; 62(5):749-57. [DOI:10.2106/00004623-19806205000008] [PMID]

[2] Spanjaard M, Reeves ND, van Dieen JH, Baltzopoulos V, Maganaris $\mathrm{CN}$. Gastrocnemius muscle fascicle behavior during stair negotiation in humans. Journal of Applied Physiology. 2007; 102(4):1618-23. [DOI:10.1152/japplphysiol.00353.2006] [PMID]

[3] McFadyen BJ, Winter DA. An integrated biomechanical analysis of normal stair ascent and descent. Journal of Biomechanics. 1988; 21(9):73344. [DOI:10.1016/0021-9290(88)90282-5]

[4] Silverman AK, Neptune RR, Sinitski EH, Wilken JM. Whole-body angular momentum during stair ascent and descent. Gait \& Posture. 2014; 39(4):1109-14. [DOI:10.1016/j.gaitpost.2014.01.025] [PMID]

[5] McCrory JL, Chambers AJ, Daftary A, Redfern MS. Ground reaction forces during stair locomotion in pregnant fallers and non-fallers. Clinical Biomechanics. 2014; 29(2):143-8. [DOI:10.1016/j.clinbioo mech.2013.11.020] [PMID]

[6] Bergland A, Sylliaas H, Jarnlo GB, Wyller TB. Health, balance, and walking as correlates of climbing steps. Journal of Aging and Physical Activity. 2008; 16(1):42-52. [DOI:10.1123/japa.16.1.42] [PMID]

[7] Conway Z, Silburn PA, Blackmore T, Cole MH. Evidence of compensatory joint kinetics during stair ascent and descent in Parkinson's disease. Gait \& Posture. 2017; 52:33-9. [DOI:10.1016/j.gaitpost.2016.11.017] [PMID]

[8] Hicks-Little CA, Peindl RD, Fehring TK, Odum SM, Hubbard TJ, Cordova $\mathrm{ML}$. Temporal-spatial gait adaptations during stair ascent and descent in patients with knee osteoarthritis. The Journal of Arthroplasty. 2012; 27(6):1183-9. [DOI:10.1016/j.arth.2012.01.018] [PMID]

[9] Meyer CA, Corten K, Fieuws S, Deschamps K, Monari D, Wesseling M, et al. Evaluation of stair motion contributes to new insights into hip osteoarthritis-related motion pathomechanics. Journal of Orthopaedic Research. 2016; 34(2):187-96. [DOI:10.1002/jor.22990] [PMID]

[10] Singh T, Koh M. Lower limb dynamics change for children while walking with backpack loads to modulate shock transmission to the head. Journal of Biomechanics. 2009; 42(6):736-42. [DOI:10.1016/j.jbioe mech.2009.01.035] [PMID]

[11] Müller R, Bisig A, Kramers I, Stüssi E. Influence of stair inclination on muscle activity in normals. Journal of Biomechanics. 1998; 31(Suppl. 1):32. [DOI:10.1016/S0021-9290(98)80067-5]

[12] Heinlein B, Kutzner I, Graichen F, Bender A, Rohlmann A, Halder AM, et al. ESB clinical biomechanics award 2008: Complete data of total knee replacement loading for level walking and stair climbing measured in vivo with a follow-up of 6-10 months. Clinical Biomechanics. 2009; 24(4):315-26. [DOI:10.1016/j.clinbiomech.2009.01.011] [PMID]

[13] Kuriki H, Azevedo F, Alves N. Characterization of biomechanical parameters during stair ascent. Journal of Biomechanics. 2012; 45(Suppl. 1):S236. [DOI:10.1016/S0021-9290(12)70237-3]

[14] Larsen AH, Puggaard L, Hämäläinen U, Aagaard P. Comparison of ground reaction forces and antagonist muscle coactivation during stair walking with ageing. Journal of Electromyography and Kinesiology. 2008; 18(4):568-80. [DOI:10.1016/j.jelekin.2006.12.008] [PMID]

[15] Aldridge JM, Sturdy JT, Wilken JM. Stair ascent kinematics and kinetics with a powered lower leg system following transtibial amputation. Gait
\& Posture. 2012; 36(2):291-5. [DOI:10.1016/j.gaitpost.2012.03.013] [PMID]

[16] Alimusaj M, Fradet L, Braatz F, Gerner HJ, WolfSI. Kinematics and kinetics with an adaptive ankle foot system during stair ambulation of transtibial amputees. Gait \& Posture. 2009; 30(3):356-63. [DOI:10.1016/j. gaitpost.2009.06.009] [PMID]

[17] Bjerke J, Öhberg F, Nilsson KG, Stensdotter AK. Compensatory strategies for muscle weakness during stair ascent in subjects with total knee arthroplasty. The Journal of Arthroplasty. 2014; 29(7):1499-502. [DOI:10.1016/j.arth.2014.01.033] [PMID]

[18] Murley GS, Menz HB, Landorf KB. Foot posture influences the electromyographic activity of selected lower limb muscles during gait. Journal of Foot and Ankle Research. 2009; 2:35. [DOI:10.1186/1757-1146-2-35] [PMID] [PMCID]

[19] Bolgla LA, Malone TR, Umberger BR, Uhl TL. Reliability of electromyographic methods used for assessing hip and knee neuromuscular activity in females diagnosed with patellofemoral pain syndrome. Journal of Electromyography and Kinesiology. 2010; 20(1):142-7. [DOI:10.1016/j. jelekin.2008.11.008] [PMID]

[20] Simpson KM, Munro BJ, Steele JR. Backpack load affects lower limb muscle activity patterns of female hikers during prolonged load carriage. Journal of Electromyography and Kinesiology. 2011; 21(5):782-8. [DOI:10.1016/j.jelekin.2011.05.012] [PMID]

[21] Brechter JH, Powers CM. Patellofemoral joint stress during stair ascent and descent in persons with and without patellofemoral pain. Gait \& Posture. 2002; 16(2):115-23. [DOI:10.1016/S0966-6362(02)00090-5]

[22] Sacco IC, Sartor CD, Cacciari LP, Onodera AN, Dinato RC, Pantaleão Jr $E$, et al. Effect of a rocker non-heeled shoe on EMG and ground reaction forces during gait without previous training. Gait \& Posture. 2012; 36(2):312-5. [DOI:10.1016/j.gaitpost.2012.02.018] [PMID]

[23] Nigg BM, Stefanyshyn D, Cole G, Stergiou P, Miller J. The effect of material characteristics of shoe soles on muscle activation and energy aspects during running. Journal of Biomechanics. 2003; 36(4):569-75 [DOI:10.1016/S0021-9290(02)00428-1]

[24] Spaepen AJ, Vanlandewijck YC, Lysens RJ. Relationship between energy expenditure and muscular activity patterns in handrim wheelchair propulsion. International Journal of Industrial Ergonomics. 1996; 17(2):163-73. [DOI:10.1016/0169-8141(95)00047-X] 